\title{
Mutation and Loss of Expression of ARID1A in Uterine Low-grade Endometrioid Carcinoma
}

\author{
Bin Guan, PhD,* Tsui-Lien Mao, MD, † Pradeep K. Panuganti, MD, * Elisabetta Kuhn, MD,* \\ Robert J. Kurman, MD, ${ }^{*}$ Daichi Maeda, MD, PhD, $\ddagger$ Elizabeth Chen, * Yung-Ming Jeng, MD, $\dagger$ \\ Tian-Li Wang, PhD,* and Ie-Ming Shih, MD, PhD*
}

\begin{abstract}
A R I D 1 A$ is a recently identified tumor suppressor gene that is mutated in approximately $50 \%$ of ovarian clear cell and $30 \%$ of ovarian endometrioid carcinomas. The mutation is associated with loss of protein expression as assessed by immunohistochemistry. In this study, we evaluated $A R I D 1 A$ immunoreactivity in a wide variety of carcinomas to determine the prevalence of $A R I D I A$ inactivation in carcinomas. Mutational analysis of $A R I D 1 A$ was carried out in selected cases. Immunoreactivity was not detected (corresponding to inactivation or mutation of $A R I D 1 A)$ in $36(3.6 \%)$ of 995 tumors. Uterine low-grade endometrioid carcinomas showed a relatively high-frequency loss of $A R I D 1 A$ expression, as 15 (26\%) of 58 cases were negative. The other tumor that had a relatively highfrequency loss of $A R I D 1 A$ expression was gastric carcinoma $(11 \%)$. Mutational analysis showed $10(40 \%)$ of 25 uterine endometrioid carcinomas; none of 12 uterine serous carcinomas and none of 56 ovarian serous and mucinous carcinomas harbored somatic ARIDIA mutations. All mutations in endometrioid carcinomas were nonsense or insertion/deletion mutations, and tumors with ARIDIA mutations showed complete loss or clonal loss of ARIDIA expression. In conclusion, this study is the first large-scale analysis of a wide variety of carcinomas showing that uterine low-grade endometrioid carcinoma is the predominant tumor type harboring ARID1A mutations and frequent loss of ARIDIA expression. These findings suggest that the molecular pathogenesis of lowgrade uterine endometrioid carcinoma is similar to that of ovarian low-grade endometrioid and clear cell carcinoma, tumors that have previously been shown to have a highfrequency loss of expression and mutation of $A R I D 1 A$.
\end{abstract}

From the Departments of *Pathology, Oncology and Gynecology and Obstetrics, Johns Hopkins University School of Medicine, Baltimore, MD; $\dagger$ Department of Pathology, National Taiwan University College of Medicine, Taipei, Taiwan; and $\$$ Department of Pathology, Graduate School of Medicine, the University of Tokyo, Tokyo, Japan.

Supported by NIH/NCI grants, CA129080, CA103937, and CA116184. B.G. is partly supported by the grant, 2010HREAOSB1, from the HERA Women's Cancer Foundation.

Bin Guan and Tsui-Lien Mao have contributed equally.

Correspondence: Ie-Ming Shih, MD, PhD, Department of Pathology and Oncology, Johns Hopkins University School of Medicine, Baltimore, Maryland (e-mail: ishih@jhmi.edu).

Copyright (C) 2011 by Lippincott Williams \& Wilkins
Key Words: ARID1A, BAF250, uterine carcinoma, ovarian carcinoma

(Am J Surg Pathol 2011;35:625-632)

A cquisition of somatic mutations is a molecular hallmark of neoplasia. Sequence mutations that are acquired during tumor evolution can lead to activation of oncogenes and inactivation of tumor suppressor and DNA repair genes, thereby propelling tumor development and progression. ${ }^{8}$ Identification and characterization of somatic mutations are not only fundamental in understanding the molecular pathogenesis of cancer but also can provide the rationale for the development of personalized diagnostic tests and therapy. With the use of whole exome sequencing and transcriptome sequencing, 2 independent studies recently reported ARIDIA (also known as $B A F 250 A$ ) mutations in $43 \%$ to $56 \%$ of ovarian clear cell carcinomas and $30 \%$ of ovarian lowgrade endometrioid carcinomas ${ }^{6,21}$ but not in matched controls, confirming the somatic nature of the mutations. As both these tumor types are believed to be derived from endometriosis and because 1 of these studies also found ARIDIA mutations in adjacent atypical endometriosis, it is conceivable that $A R I D 1 A$ loss is a relatively specific molecular event in the genesis of these tumors. Many ARIDIA mutations are insertion/deletion mutations, leading to the generation of premature stop codons by frameshift that result in truncated proteins prone to degradation. It has been previously shown that loss of $A R I D 1 A$ expression, as assessed by immunohistochemistry (IHC), correlates closely with ARIDIA mutations. ${ }^{11,21}$

ARIDIA is located in the chromosome $1 \mathrm{p} 36$ region, and encodes a large nuclear protein involved in chromatin remodeling. ARIDIA interacts with several other proteins including the core protein, BRG or BRM with ATPase activity. ${ }^{4,20}$ The $A R I D 1 A$-BRG/BRM complex belongs to the SWI/SNF chromatin remodeling complex; remodeling activity is facilitated by ATP hydrolysis of BRG or BRM. In contrast, the noncatalytic subunits of the SWI/ SNF complex, such as ARID1A, are responsible for modulating the target specificity and activity of the ATPase. The chromatin remodeling activity of SWI/SNF 
has been shown to play an integral role in controlling gene expression ${ }^{19}$ and is critical in tissue development, cellular differentiation, and tumor suppression. ${ }^{3,4,15} A R$ $I D 1 A$ is essential for $\mathrm{SWI} / \mathrm{SNF}$ complexes to suppress DNA synthesis. Inactivation of ARIDIA is thought to enhance cell cycle progression by potentially involving c-myc, thereby contributing to uncontrolled cellular proliferation in cancer cells. $., 13,14$

Although ARIDIA has emerged as a new cancerassociated gene, which is frequently mutated in endometriosis-related ovarian neoplasms, it is not known whether its mutation, such as $F O X L 2^{10,17}$ and $A P C,{ }^{9}$ is detected only in specific types of cancer or mutations, such as $T P 53$ and KRAS, which occurs in a variety of neoplastic diseases. As ARIDIA mutations are randomly distributed in 20 exons and are the insertion/deletion type of mutations that lead to truncated proteins, we used loss of ARIDIA immunoreactivity as a surrogate marker for a mutation to screen a variety of carcinomas. Sequence analysis was then carried out in the specimens that showed the highest frequency of loss of ARIDIA expression.

\section{MATERIALS AND METHODS}

\section{Tissue Material}

Paraffin-embedded tissue sections of normal and tumor tissues from various organs were obtained from the Department of Pathology of the National Taiwan University Hospital and Johns Hopkins Hospital, from 1994 to 2009. The normal tissues studied by IHC included esophagus, stomach, colon, salivary gland, liver, pancreas, lung, kidney, prostate, adrenal gland, testis, breast, thyroid, tonsil, and placenta. The tumors included 41 hepatocellular carcinomas, 27 bile duct carcinomas, 52 pulmonary carcinomas (42 adenocarcinomas, 10 squamous carcinomas), 73 renal cell carcinomas, 91 breast invasive ductal carcinomas, 272 ovarian tumors (221 high-grade serous carcinomas, 15 low-grade serous carcinomas, and 36 mucinous carcinomas), 58 trophoblastic tumors (35 choriocarcinomas, 6 placental site trophoblastic tumors, 17 epithelioid trophoblastic tumors), 125 cervical carcinomas (114 squamous cell carcinomas, 11 adenocarcinomas), 75 uterine carcinomas (58 conventional low-grade endometrioid carcinomas, 15 serous carcinomas, 2 carcinosarcomas), 35 prostate carcinomas, 49 colon carcinomas, 45 gastric carcinomas, 48 pancreatic carcinomas, and 4 oral squamous cell carcinomas. The use of the archival materials was approved by the internal review board of both institutions.

For mutation analysis, genomic DNA isolated from affinity purified tumor samples was used. Those samples included 25 uterine endometrioid carcinomas (International Federation of Gynecology and Obstetrics grade 1), 12 uterine serous carcinomas, 32 ovarian high-grade serous carcinomas, 19 ovarian low-grade serous carcinomas, and 5 ovarian mucinous carcinomas. As the ARIDIA mutation status has been previously reported in ovarian clear cell and ovarian low-grade endometrioid carcinomas, ${ }^{6,11,21}$ these carcinomas were not included in this study. The methodology for the isolation of tumor cells from fresh carcinoma specimens has been previously described. ${ }^{6}$

\section{IHC}

IHC analysis was carried out on tissue microarrays except for 20 uterine endometrioid carcinomas and 33 renal cell carcinomas, which were carried out on wholetissue sections. Loss of ARIDIA expression detected in the tissue microarrays was confirmed on whole-tissue sections. A polyclonal rabbit anti-ARIDIA antibody (Sigma-Aldrich HPA005456) was generated by immunizing a rabbit with the following peptide sequence: PGLGNVAMGPRQHYPYGGPYDRVRTEPGIGPEG NMSTGAPQPNLMPSNP DSGMYSPSRYPPQQQQQQ QQRHDSYGNQFSTQGTPSGSPFPSQQTTMYQQQQ QNYK. The specificity of the antibody was confirmed by Western blotting. Antigen retrieval was performed by placing sections in a citrate buffer $(\mathrm{pH}$ 6.0), which were then placed in an autoclave at $120^{\circ} \mathrm{C}$ for 10 minutes. The sections were incubated with the rabbit antibody overnight at $4{ }^{\circ} \mathrm{C}$. A positive reaction was detected by the EnVision + System (Dako, Carpinteria, CA). Tumor stromal cells served as positive internal controls. Only nuclear staining was scored. A previous study showed that loss of nuclear expression correlated with mutation of the gene. Hence, absence of nuclear staining (diffuse or focal) was considered to be positive for gene mutation.

\section{Mutation Analysis}

A total of 93 tumor samples were analyzed for somatic ARIDIA mutations. The normal tissues from the matched cases were also sequenced in parallel. Nucleotide sequences of polymerase chain reaction primers that amplified exon 1 to exon 20 were previously reported. ${ }^{6}$ Polymerase chain reaction products were prepared and purified for Sanger sequencing.

\section{Gene Knockdown and Western Blot}

The lentivirus-expressing ARIDIA short hairpin ribonucleic acids (shRNAs) were produced using HEK293FT cells transfected with the pLKO.1-puro lentiviral plasmids (the RNAi consortium) and the second generation packaging system, pSPAX2 (Addgene plasmid 12260) and pMD2.G (Addgene plasmid 12259). The shRNA sequences were: shRNA1, GCCTGATCTATCT GGTTCAAT; shRNA2, CCTCTCTTATACACAGCA GAT; and shRNA3, CCGTTGATGAACTCATTGGTT. HeLa cells were transduced with lentiviral particles, and lysates in Laemmli sample buffer were prepared from the cells 3 days after transduction. For Western blots, SDSPAGE was used to separate proteins that were then transferred onto PVDF membranes. The rabbit antiARIDIA antibody as used in IHC was used to hybridize the membranes (at a dilution of 1:2000), and antiGAPDH antibody was also applied to detect the GAPDH, serving as the loading control. After incubating 
at room temperature for 2 hours, the membranes were washed with TBST $(0.01 \%$ Tween 20 in tris-buffered solution) and were blotted with HRP-conjugated antirabbit antibodies (Pierce, Rockford, IL) at a dilution of 1:1000 for 1 hour at room temperature. ARIDIA and GAPDH bands were shown by chemiluminescence (Arlington Heights, IL).

\section{RESULTS}

A total of 995 carcinomas from a variety of tissue origins were studied for $A R I D 1 A$ expression using IHC. To confirm the specificity of the anti-ARIDIA antibody used in this study, we performed a gene knockdown experiment by transducing HeLa cells with 3 different ARIDIA shRNAs. Western blot analysis showed a significant decrease of $A R I D I A$ protein in HeLa cells after treatment with $A R I D 1 A$-specific shRNAs, especially the shRNA-2 and shRNA-3 compared with control shRNA, indicating the specificity of the ARIDIA antibody (Fig. 1). Previous studies have shown that inactivating mutations of ARIDIA are associated with loss of protein expression..$^{1,21}$ Therefore, we focused our attention on those tumors with undetectable ARIDIA immunoreactivity, and used a scoring system to classify all cases into ARID IA-negative (undetectable) and ARIDIApositive cases, which showed any levels of ARIDIA immunoreactivity. As the ARIDIA mutation status has been previously reported in ovarian clear cell and ovarian endometrioid carcinomas, ${ }^{6,11,21}$ these carcinomas were not included in this study.

A

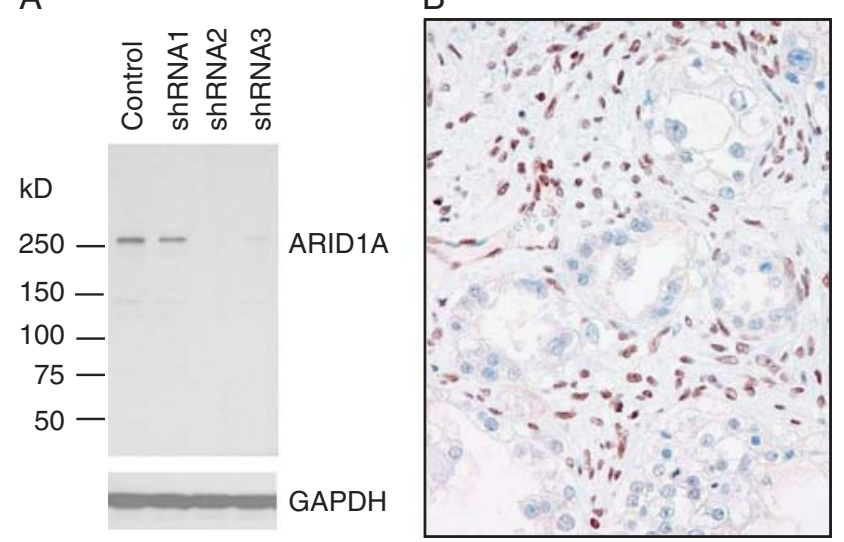

FIGURE 1. Specificity of the antibody in detecting ARIDIA. Western blot analysis shows a predominant protein band, with a molecular mass corresponding to ARID1A protein (approximately $280 \mathrm{kD}$ ) in HeLa cell lysate. Protein expression is significantly decreased in shRNA-2-treated and shRNA-3treated HeLa cells compared with control shRNA-treated cells (A). IHC using this anti-ARIDIA antibody on an ovarian clear cell carcinoma with known biallelic somatic insertion/deletion mutations of ARID1A (B). The tumor cells show undetectable ARID1A immunoreactivity, whereas the stromal cells show intense nuclear staining.

\begin{tabular}{|c|c|c|c|}
\hline$\underline{\text { Tumor }}$ & $\begin{array}{c}\text { Total Case } \\
\text { No. } \\
\end{array}$ & IHC $^{-}$ & $\begin{array}{c}\text { Percentage } \\
\text { of IHC }\end{array}$ \\
\hline Hepatocellular carcinoma & 41 & 0 & 0 \\
\hline Bile duct carcinoma & 27 & 2 & 7.4 \\
\hline \multicolumn{4}{|l|}{ Lung } \\
\hline Adenocarcinoma & 42 & 1 & 2.4 \\
\hline Squamous carcinoma & 10 & 1 & 10 \\
\hline Renal cell carcinoma & 73 & 0 & 0 \\
\hline Breast carcinoma & 91 & 1 & 1.1 \\
\hline \multicolumn{4}{|l|}{ Ovary } \\
\hline High-grade serous carcinoma & 221 & 0 & 0 \\
\hline Low-grade serous carcinoma & 15 & 0 & 0 \\
\hline Mucinous carcinoma & 36 & 0 & 0 \\
\hline \multicolumn{4}{|l|}{ Uterine cervix } \\
\hline Squamous carcinoma & 114 & 2 & 1.8 \\
\hline Adenocarcinoma & 11 & 1 & 9.1 \\
\hline \multicolumn{4}{|l|}{ Uterine corpus } \\
\hline Endometrioid carcinoma & 58 & 15 & 26 \\
\hline Serous carcinoma/carcinosarcoma & 17 & 0 & 0 \\
\hline \multicolumn{4}{|l|}{ Trophoblastic tumor } \\
\hline Choriocarcinoma & 35 & 0 & 0 \\
\hline Placental site trophoblastic tumor & 6 & 0 & 0 \\
\hline Epithelioid trophoblastic tumor & 17 & 0 & 0 \\
\hline Prostate carcinoma & 35 & 0 & 0 \\
\hline Colon carcinoma & 49 & 2 & 4.1 \\
\hline Gastric carcinoma & 45 & 5 & 11 \\
\hline Pancreatic carcinoma & 48 & 4 & 8.3 \\
\hline Oral squamous carcinoma & 4 & 0 & 0 \\
\hline
\end{tabular}

There was loss of expression of ARID1A in 34 $(3.4 \%)$ of 995 cases. Stromal cells in these cases were positive for ARIDIA immunoreactivity, indicating that the negative staining in tumor cells was not due to technical artifacts. The immunostaining findings for each tumor type are summarized in Table 1 and representative cases illustrated in Figure 2. Specifically, among 21 types of carcinoma in this study, uterine low-grade endometrioid carcinomas showed loss of expression in 15 (26\%) of 58 cases. In contrast, all the normal endometrial tissues examined in this study together with 38 normal endometrial tissues analyzed in our previous study ${ }^{11}$ were intensely positive for $A R I D I A$. In addition to uterine lowgrade endometrioid carcinomas, gastric carcinomas were negative for $A R I D 1 A$ expression in $11 \%$ of cases. We also observed that some carcinomas, especially uterine lowgrade endometrioid carcinomas, exhibited "clonal loss" of ARIDIA immunoreactivity. This means that in a background of $A R I D I A$-positive tumor cells, large groups of tumor cells did not express ARIDIA (Fig. 3). Although several other tumor types including carcinomas of the bile duct, lung, breast, uterine cervix, colon, and pancreas contained at least 1 case with negative ARIDIA staining, the frequency of negative cases was very low $(<10 \%$ of cases in each tumor type). In contrast, ARID $1 A$ was expressed in virtually all the epithelial cells in all normal adult and embryonic tissues tested which included breast, prostate, gastrointestinal tract, pancreas, bile duct, mullerian duct including endometrium (both premenopausal and postmenopausal), skin, respiratory tract, urinary tract, and trophoblast. In addition, lymphoblasts 

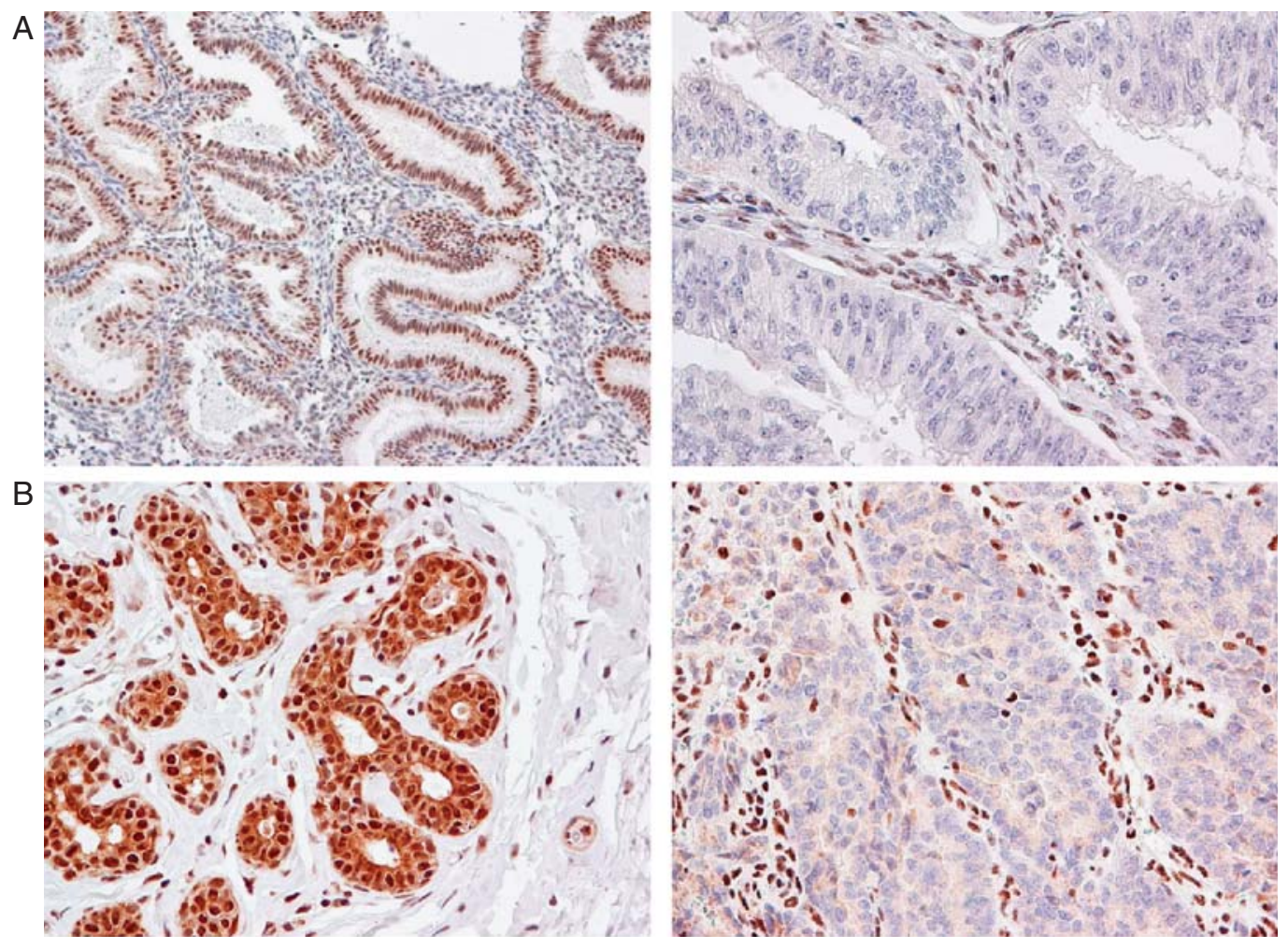

C
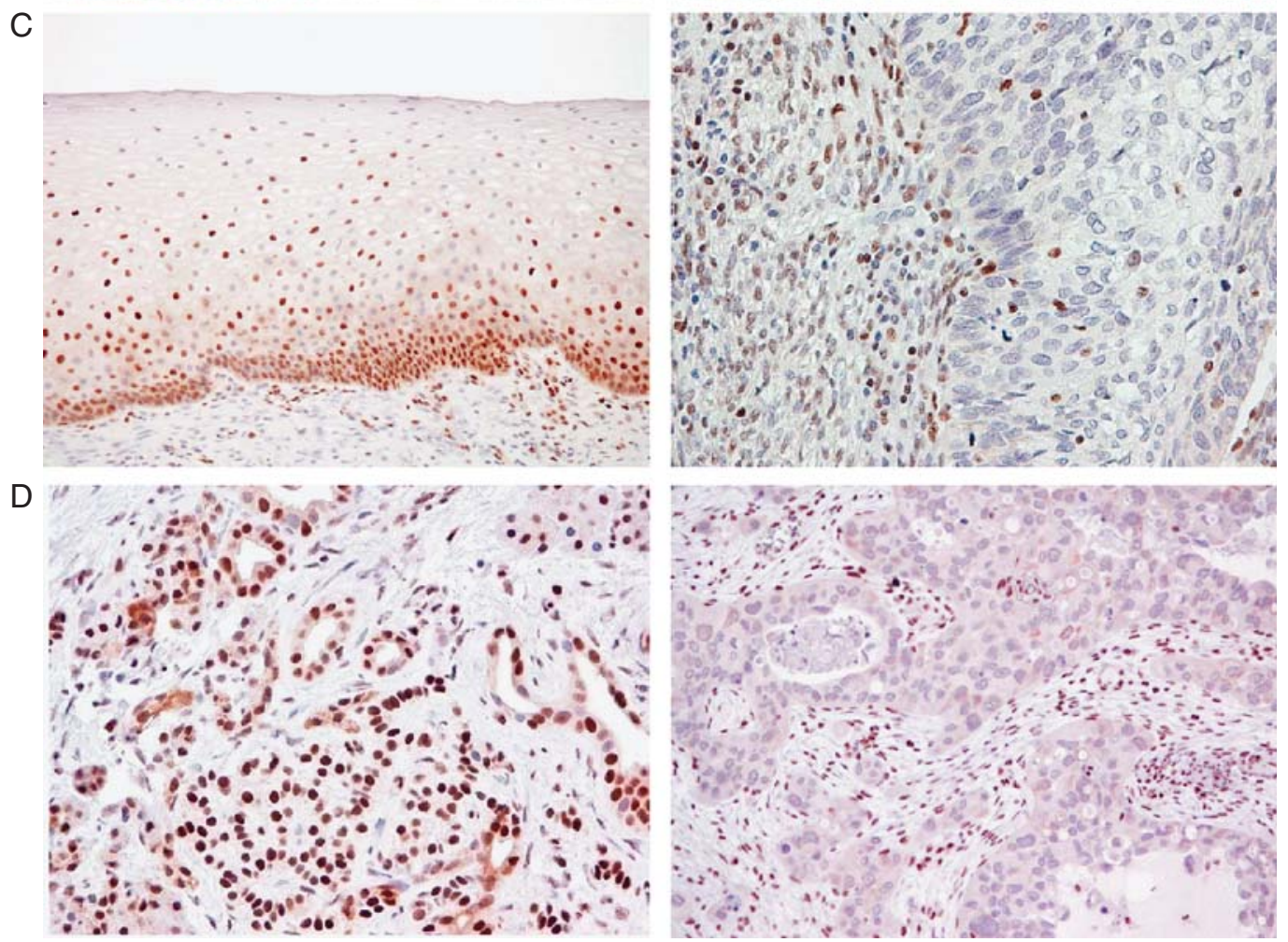

FIGURE 2. ARID1A immunoreactivity in representative carcinoma types (right panel) and their normal tissue counterparts (left panel). Negative staining (undetectable level) of ARID1A in an International Federation of Gynecology and Obstetrics grade I endometrioid carcinoma (A), an infiltrating ductal carcinoma of the breast (B), a cervical squamous carcinoma (C), and a pancreatic carcinoma (D). 

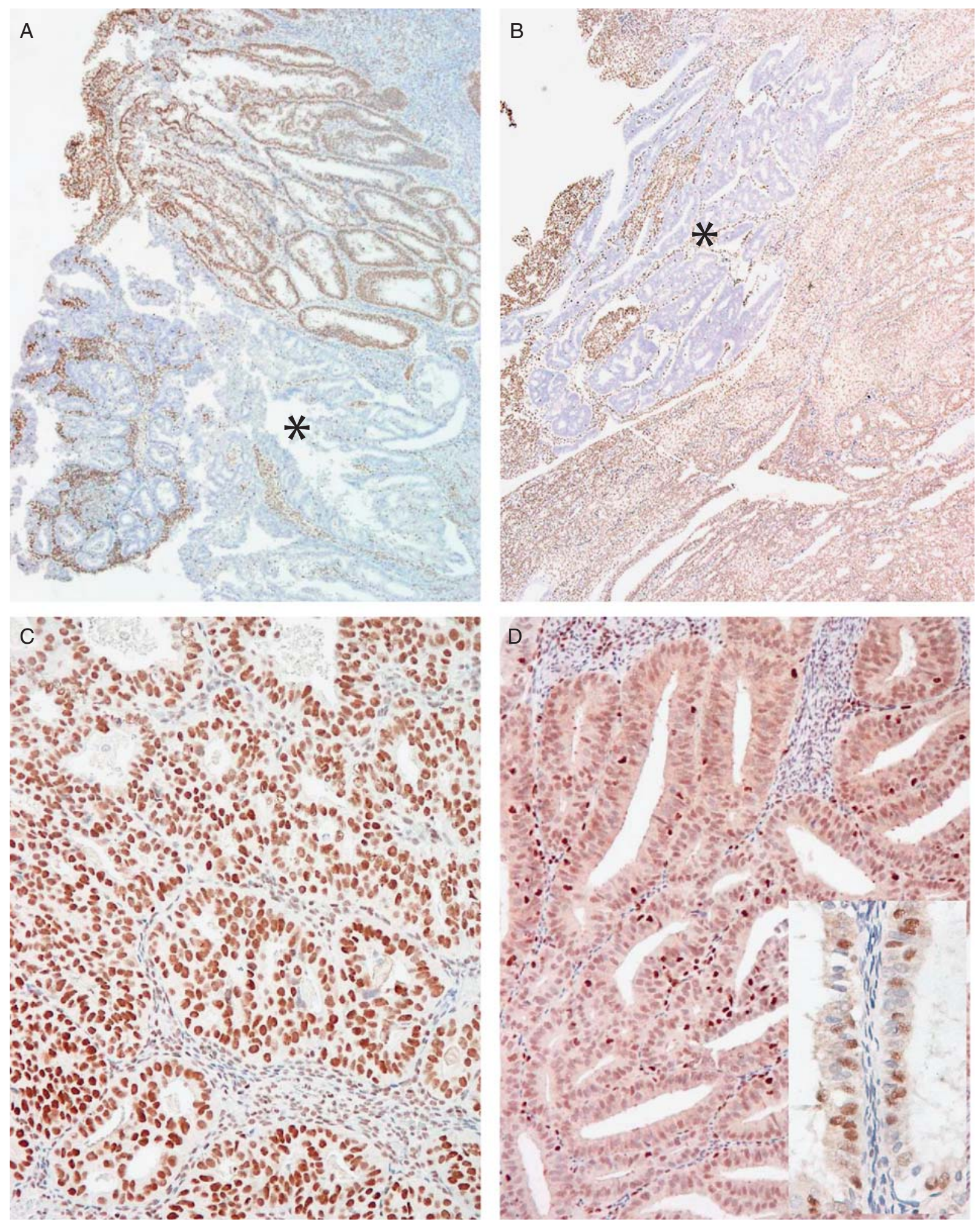

FIGURE 3. Pattern of ARID1A immunoreactivity in 4 uterine endometrioid carcinomas. A, The carcinoma (UEM-5) harbors biallelic ARID1A mutations (nonsense and deletion mutation) and shows a clonal loss of ARID1A expression (asterisk). B, The carcinoma (UEM-3) containing monoallelic nonsense mutation shows a clonal loss of ARID1A expression (asterisk). C, The carcinoma with wild-type $A R I D 1 A$ exhibits a diffuse and intense pattern of $A R I D 1 A$ staining. D, The carcinoma with wild-type $A R I D 1 A$ shows diffuse but less intense ARID1A immunoreactivity than the tumor in C. Occasionally, patchy staining can be observed (inset). 
TABLE 2. ARID1A Somatic Mutations in Uterine and Ovarian Carcinomas

\begin{tabular}{|c|c|c|c|c|c|}
\hline & Uterine Endometrioid & Uterine Serous & Ovarian HG & Ovarian LG & Ovarian Mucinous \\
\hline Cases with $A R I D 1 A$ mutation & 10 & 0 & 0 & 0 & 0 \\
\hline Total cases & 25 & 12 & 32 & 19 & 5 \\
\hline
\end{tabular}

HG indicates high grade; LG, low grade.

in the germinal center, smooth muscle cells, skeletal muscle cells, and endothelial cells were also positive for ARID1A.

Mutational analysis was carried out on uterine low-grade endometrioid carcinomas because they showed the highest frequency of loss of ARIDIA expression. Nineteen ovarian low-grade, 32 ovarian high-grade, 5 ovarian mucinous, and 12 uterine serous carcinomas were also analyzed, none of which showed loss of ARIDIA expression. As shown in Table 2, somatic ARIDIA mutation was detected in $10(40 \%)$ of 25 uterine lowgrade endometrioid carcinomas. As in ovarian clear cell and ovarian endometrioid carcinomas, the mutations were either insertion/deletion mutations or nonsense mutations that were widely distributed in the ARIDIA gene (Table 3). Three of these 10 cases (UEM-1, UEM-5, and UEM-8) showed 2 independent $A R I D I A$ mutations, likely affecting both alleles. Correlation of ARIDIA mutation status and immunoreactivity was made in 25 uterine endometrioid carcinomas and 51 ovarian serous carcinomas. We found that $5(50 \%)$ of 10 tumors with $A R I D 1 A$ mutations did not show any detectable level of ARIDIA immunoreactivity. Interestingly, 4 ARIDIApositive cases with ARIDIA mutations exhibited a pattern of immunoreactivity in which areas of negative cells were present adjacent to positive areas suggesting that mutations arose in clones within the tumor (Fig. 3). In contrast, only 2 of $15 A R I D 1 A$ wild-type endometrioid carcinomas showed complete loss of ARIDIA staining, whereas the majority of cases showed diffuse ARIDIA staining ( $>80 \%$ of tumor cells being positive). We did not observe any pattern of clonal loss in ARIDIA wildtype carcinomas as in ARIDIA-mutated cases. All 19 ovarian low-grade serous carcinomas and 32 ovarian high-grade serous carcinomas showed diffuse ARIDIA positivity; neither the above-mentioned carcinomas nor the 5 ovarian mucinous carcinomas contained ARIDIA mutations (Table 2). Thus, the complete loss of ARID1A expression significantly correlated with its mutation status $(P=0.0014$, Fisher exact test). Combining cases that were completely negative with those showing clonal loss and correlating them with $A R I D I A$ mutation was found to be highly significant $(P<0.0001$, Fisher exact test $)$.

\section{DISCUSSION}

In the past few years, substantial progress has been made in cataloging molecular genetic alterations at a genome-wide scale. One of the main findings has been the identification of somatic mutations of several chromatin- remodeling genes in certain types of human cancer. These genes include $J A R I D 1 C$ in renal cell carcinoma, ${ }^{1} B R G 1$ (SMARCA4) in lung carcinoma, ${ }^{12,16}$ and, most recently, $A R I D 1 A$ in ovarian clear cell and ovarian low-grade endometrioid carcinoma. ${ }^{6,21}$ The findings in this study extend previous observations and provide cogent evidence that epigenetic changes, like genetic alteration, is a "driver" rather than a "passenger" that is directly involved in the tumor development of uterine low-grade endometrioid carcinoma. ${ }^{7}$

In this study, a large number and variety of normal and tumor tissues were evaluated. The main findings were the loss of ARIDIA immunoreactivity and mutation of $A R D I A$ in low-grade uterine endometrioid carcinoma compared with other types of carcinomas. Although loss of ARIDIA expression also occurs in other tumor types, the frequency of loss of expression is low, indicating that $A R I D 1 A$ mutations are associated with specific types of carcinoma. Mutational analysis showed somatic ARIDIA mutations in $40 \%$ of uterine endometrioid carcinoma, a finding that has not been previously reported.

Endometrial carcinomas are divided into 2 broad groups, designated as type I and type II. Type I tumors are composed of endometrioid carcinomas that frequently harbor sequence mutations in CCNB1, PTEN, and PIK3CA, whereas type II tumors are largely serous carcinomas that contain TP53 mutations in the majority of cases. ${ }^{2}$ Patients with type I tumors are usually younger, present at an earlier clinical stage, and have a more indolent clinical course compared with women with type II tumors. Type I tumors arise from endometrial hyperplasia, and type II tumors develop from endometrial intraepithelial carcinoma that is frequently associated with endometrial polyps. Thus, the relatively frequent loss of ARIDIA expression and ARIDIA mutations in uterine endometrioid carcinoma but not in uterine serous carcinoma further supports their distinct pathogenesis. Given the well-established roles of the Pten-AKT pathway and the Wnt pathway in the development of lowgrade uterine endometrioid carcinoma (type I tumor), it will be important to determine whether the ARIDIA pathway cross talks with those signaling pathways and to assess how inactivation of $A R I D 1 A$ contributes to tumor initiation and progression in this type of carcinoma.

Although ARIDIA mutations were most often associated with complete loss of its protein expression in low-grade uterine endometrioid carcinoma, we observed several uterine endometrioid carcinomas with ARID 1A mutations that showed a heterogeneous staining pattern. In these cases, there were significantly large areas 
TABLE 3. Correlation of ARID1A Mutations and Immunoreactivity

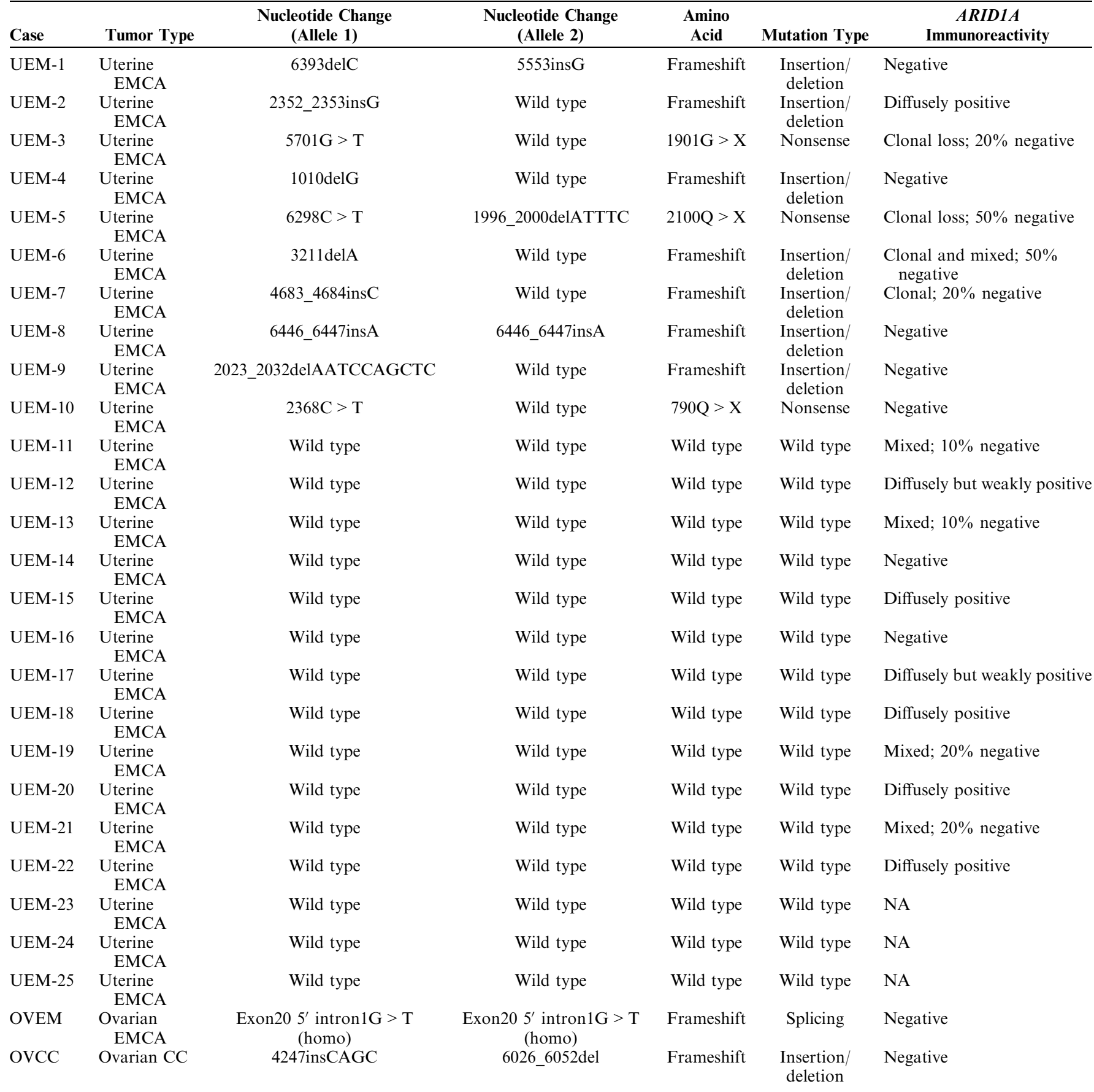

CC indicates clear cell carcinoma; EMCA, endometrioid carcinoma; NA, not applicable.

that were negative in what were otherwise positive cases. This geographic distribution of loss of $A R I D 1 A$ expression strongly suggests that $A R I D I A$ mutation occurred in clones of cells within the tumor. This type of clonal loss of $A R I D 1 A$ immunoreactivity was only detected in uterine endometrioid carcinomas with $A R I D I A$ mutations but not in those without mutations. It is likely that $A R I D I A$ mutations occur after tumor initiation in endometrioid carcinoma, creating tumor subclones during evolution of the carcinoma. It is of great interest to study $A R I D I A$ 
immunostaining patterns in endometrial hyperplasia to determine how early ARIDIA protein is lost during tumor progression of endometrioid carcinoma.

Similar to endometrial carcinoma, ovarian epithelial carcinomas have been divided into type I and type II categories based on their distinctive clinicopathologic and molecular features. ${ }^{18}$ Ovarian clear cell carcinoma and low-grade ovarian endometrioid carcinoma comprise the majority of type I ovarian tumors and are frequently associated with endometriosis. The results from this study along with our previous reports ${ }^{6,11,21}$ strongly suggest that loss of $A R I D 1 A$ expression and/or its mutations are largely confined to ovarian clear cell and ovarian endometrioid carcinomas, because the high-grade and low-grade serous carcinomas and mucinous carcinomas did not show $A R I D 1 A$ mutations or loss of expression. It is, therefore, conceivable that ARIDIA mutation plays an important role in the development of ovarian tumors derived from endometriosis. As it is generally thought that endometriosis develops from retrograde menstruation, the underlying critical molecular event for the development of uterine low-grade endometrioid, ovarian endometrioid, and clear cell carcinomas in some cases is mutation of ARIDIA in the endometrial tissue. Although we did not detect $A R I D 1 A$ mutation in ovarian mucinous carcinomas, the small number of mucinous carcinomas analyzed in this study precludes a definitive conclusion regarding them.

In conclusion, based on ARIDIA IHC and mutational analysis, we found that ARIDIA inactivation, either by somatic mutations or by loss of expression, frequently occurs in uterine low-grade endometrioid carcinomas in addition to ovarian low-grade endometrioid and ovarian clear cell carcinomas. Thus, it seems that ARIDIA inactivation is mainly confined to certain types of gynecologic cancers that arise from endometrial tissue, either from the uterine cavity or from an ectopic site, that is, endometriosis. This therefore leads to the conclusion that these endometrium-related tumors share a similar molecular pathogenesis. Our findings also support the use of complete loss or clonal loss of ARID 1A immunoreactivity as a surrogate marker to detect $A R I D 1 A$ mutations in tissues. However, further studies are necessary to determine the role of ARID $1 A$ in the differential diagnosis. It would be also interesting to determine the mutation status and immunoreactivity of ARIDIA in uterine high-grade endometrioid carcinoma and clear cell carcinoma.

\section{REFERENCES}

1. Dalgliesh GL, Furge K, Greenman C, et al. Systematic sequencing of renal carcinoma reveals inactivation of histone modifying genes. Nature. 2010;463:360-363.

2. Di Cristofano A, Ellenson LH. Endometrial carcinoma. Annu Rev Pathol. 2007;2:57-85.

3. Gao X, Tate P, Hu P, et al. ES cell pluripotency and germ-layer formation require the SWI/SNF chromatin remodeling component BAF250a. Proc Natl Acad Sci USA. 2008;105:6656-6661.

4. Ho L, Crabtree GR. Chromatin remodelling during development. Nature. 2010;463:474-484.

5. Huang J, Zhao YL, Li Y, et al. Genomic and functional evidence for an ARID1A tumor suppressor role. Genes Chromosomes Cancer. 2007:46:745-750.

6. Jones S, Wang TL, Shih IM, et al. Frequent mutations of chromatin remodeling gene ARIDIA in ovarian clear cell carcinoma. Science. 2010;330:228-231.

7. Kaiser J. Genes link epigenetics and cancer. Science. 2010;330:577.

8. Kinzler KW, Vogelstein B. Cancer-susceptibility genes. Gatekeepers and caretakers [news; comment]. Nature. 1997;386:761, 763.

9. Kinzler KW, Nilbert MC, Vogelstein B, et al. Identification of a gene located at chromosome $5 \mathrm{q} 21$ that is mutated in colorectal cancers. Science. 1991;251:1366-1370.

10. Kobel M, Gilks CB, Huntsman DG. Adult-type granulosa cell tumors and FOXL2 mutation. Cancer Res. 2009;69:9160-9162.

11. Maeda D, Mao T-L, Fukayama M, et al. Clinicopathological Significance of Loss of ARID 1A Immunoreactivity in Ovarian Clear Cell Carcinoma. Int J Mol Sci. 2010;11:5120-5128.

12. Medina PP, Romero OA, Kohno $\mathrm{T}$, et al. Frequent BRG1/ SMARCA4-inactivating mutations in human lung cancer cell lines. Hum Mutat. 2008;29:617-622.

13. Nagl NG Jr, Patsialou A, Haines DS, et al. The p270 (ARID1A/ SMARCF1) subunit of mammalian SWI/SNF-related complexes is essential for normal cell cycle arrest. Cancer Res. 2005;65:9236-9244.

14. Nagl NG Jr, Zweitzig DR, Thimmapaya B, et al. The c-myc gene is a direct target of mammalian SWI/SNF-related complexes during differentiation-associated cell cycle arrest. Cancer Res. 2006;66: 1289-1293.

15. Reisman D, Glaros S, Thompson EA. The SWI/SNF complex and cancer. Oncogene. 2009;28:1653-1668.

16. Rodriguez-Nieto S, Canada A, Pros E, et al. Massive parallel DNA pyrosequencing analysis of the tumor suppressor BRG1/SMARCA4 in lung primary tumors. Hum Mutat. 2010;32:E1999-2017.

17. Schrader KA, Gorbatcheva B, Senz J, et al. The specificity of the FOXL2 c. $402 \mathrm{C}>\mathrm{G}$ somatic mutation: a survey of solid tumors. PLoS One. 2009; 4:e7988.

18. Shih I-M, Kurman RJ. Ovarian tumorigenesis- a proposed model based on morphological and molecular genetic analysis. $\mathrm{Am} \mathrm{J}$ Pathol. 2004;164:1511-1518.

19. Van Rechem C, Boulay G, Leprince D. HICl interacts with a specific subunit of SWI/SNF complexes, ARID1A/BAF250A. Biochem Biophys Res Commun. 2009;385:586-590.

20. Wang X, Nagl NG, Wilsker D, et al. Two related ARID family proteins are alternative subunits of human SWI/SNF complexes. Biochem J. 2004:383:319-325.

21. Wiegand KC, Shah SP, Al-Agha OM, et al. ARID1A mutations in endometriosis-associated ovarian carcinomas. $N$ Engl $J$ Med. 2010;363:1532-1543. 